\title{
Vehicle Radiators' Performance Calculation and Improvement Based on the Coupling of Multi-scale Models Simulations
}

\author{
Liu Shui-Chang ${ }^{1,2}, \mathrm{Li}_{\mathrm{Li}}-\mathrm{Fu}{ }^{1}$ and Zhang Yong ${ }^{*}, 2$ \\ ${ }^{I}$ School of Mechanical and Automotive Engineering, South China University of Technology, Guangzhou 510641, China \\ ${ }^{2}$ School of Mechanical Engineering, Hunan University of Technology, Zhuzhou 412007, China
}

\begin{abstract}
In simulation of the heat transfer between radiator and air flow field, the adoption of the radiator full-size model containing its core body structure with small feature sizes would require huge storage space and not be economical. In view of this question, based on the coupling of multi-scale models simulations, a calculation method of radiator performance is proposed in this paper the reliability of which is verified by an experiment test. Subsequently, the influence on the radiators' thermal performance of the layout of the parts in front of the radiators is analyzed. Lastly, the layout of the front parts is modified to enhance the radiators' thermal performance. The investigation results indicate that: the radiators' thermal performance calculation method based on simulations coupling of radiator multi-scale models considers the influences of air-side flow field distribution and the core body structure details; the error of the calculating values from the method is less than $5 \%$, and the method is reliable; when the heat source parts in front of the radiators are situated right in front of the rear fan channel, the radiators thermal performance is better; the radiators cooling power increases $19.3 \mathrm{~kW}$ after layout modification of the front heat resource parts.
\end{abstract}

Keywords: Multi-scale models, parts layout, radiator performance calculation.

\section{INTRODUCTION}

The radiator is important for ensuring the vehicle's power and security. In actual engineering conditions, air flow fields around radiators are often non-uniform which may generate negative effect on heat dissipation. To make the radiators more reliable and economical, many researches have been carried out. Chiou investigated two arrangements of condenser and radiator in the cooling air circuit of automobile and the result showed that side-by-side arrangement requires smaller exchanger core volume and less fan air horsepower [1]. General Motors Corporation proposed condenser, fan, radiator power train cooling module (CFRM), with the heat dissipation capacity for the CFRM configuration being larger than that of CRFM (condenser, radiator, fan power train cooling module) [2]. Hak Jun and Charn-Jung using CFD tools analyzed the impacts of fan partial displacement and radiator's size on cooling module performance [3]. Juan modeled heat exchanger as porous medium using the CFD software Fluent and analyzed the influence of front end and cooling system components on flow field around heat exchanger but heat transfer was not referred [4]. Larsson et al. found that at higher speed, the air inlet has to be redesigned to obtain a more uniform airflow over the heat exchangers as well as for obtaining higher cooling performance [5]. Heinzelmann et al. investigated the closing models of heat exchanger inlet slots and introduced that closing the upper inlet slot causes a

\footnotetext{
*Address correspondence to this author at the Vehicle Engineering Institute, Hunan University of Technology, No.88, Taishan West Road, Zhu-Zhou, Hunan, 412007, China; Tel: +8615292200781;

E-mail: zhangyong7051678@163.com
}

more significant increase in the downstream fluid temperature than closing the lower inlet slot [6]. Saab et al. analyzed the impact of the underhood opening area on the cooling air speed [7] and Mao et al. compared the heat exchanger performance of different air velocity distributions [8].

The heavy mining dump truck has the merits of heavy carrying capacity, high efficiency and low operating costs,. It takes on the $40 \%$ of the coal and $80 \%$ of the iron ore transport task in the world $[9,10]$. The cooling loads of the radiators in heavy mining dump truck are much larger than the general car but the running velocity of the mining dump truck is much lower than the general car. So, efficient radiators are particularly important for the mining dump truck to work safely and steadily. The purpose of this paper is to calculate and improve the thermal performance of the radiators positioned in a separated air channel of a mining dump truck, as shown in Figs. $(\mathbf{1}, \mathbf{2})$. Considering the air flow field distribution's influence, the simulation of 3 dimensions (3D) air flow field around radiators is necessary for carrying out radiator performance calculation. The feature size magnitude of the radiator core body is about two times smaller than that of the radiator outline, so in simulations, the radiator's full-size model requires huge storage space which is hard to accommodate. In order to meet this requirement, radiator models of multi-scale levels are established and based on the simulations coupling of the multi-scale models, the performance calculation of radiators is carried out. The performance calculation method reliability is verified by an experiment test of the mining dump truck. Then, the influence on the radiators' thermal performance of the layout of the heat source parts in front of 
the radiators is analyzed. At last, the layout of the parts is modified to improve the radiators' thermal performance.

\section{CHANNEL PROFILE AND RADIATORS}

Cooling module of the mining dump truck is positioned in a separated air channel as shown in Fig. (1). There are five radiators in it. The two bigger radiators (front radiator and back radiator) are arranged in series and their performance calculation and improvement are the main objectives in the next paragraph. The three small radiators are respectively hydraulic oil radiator, fuel oil radiator and air conditioning condenser and they are arranged side-by-side in front of the front radiator.

The two bigger radiators are ribbon-tubular radiators with the core body composed of the same size flat tube (water pipe) and corrugated ribbon. The partial enlarged drawing for the ribbon-tubular radiator is shown in Fig. (2). A water pipe matches two corrugated ribbons. Heat fluid water flows in the water pipe and cooling air crosses the corrugated ribbon and the external of water pipe. The core body of the front radiator includes two rows of water pipes and the back radiator includes three.

\section{RADIATORS' THERMAL PERFORMANCE CALCULATION AND VERIFICATION}

\subsection{Physics Equations of Flow Field}

Rated operating condition of the mining dump truck is selected for investigation in this paper. The running velocity of the truck is $3.33 \mathrm{~m} / \mathrm{s}$ and fan rotating speed is $1000 \mathrm{r} / \mathrm{min}$. So the Mach number of the radiator air-side flow field in the

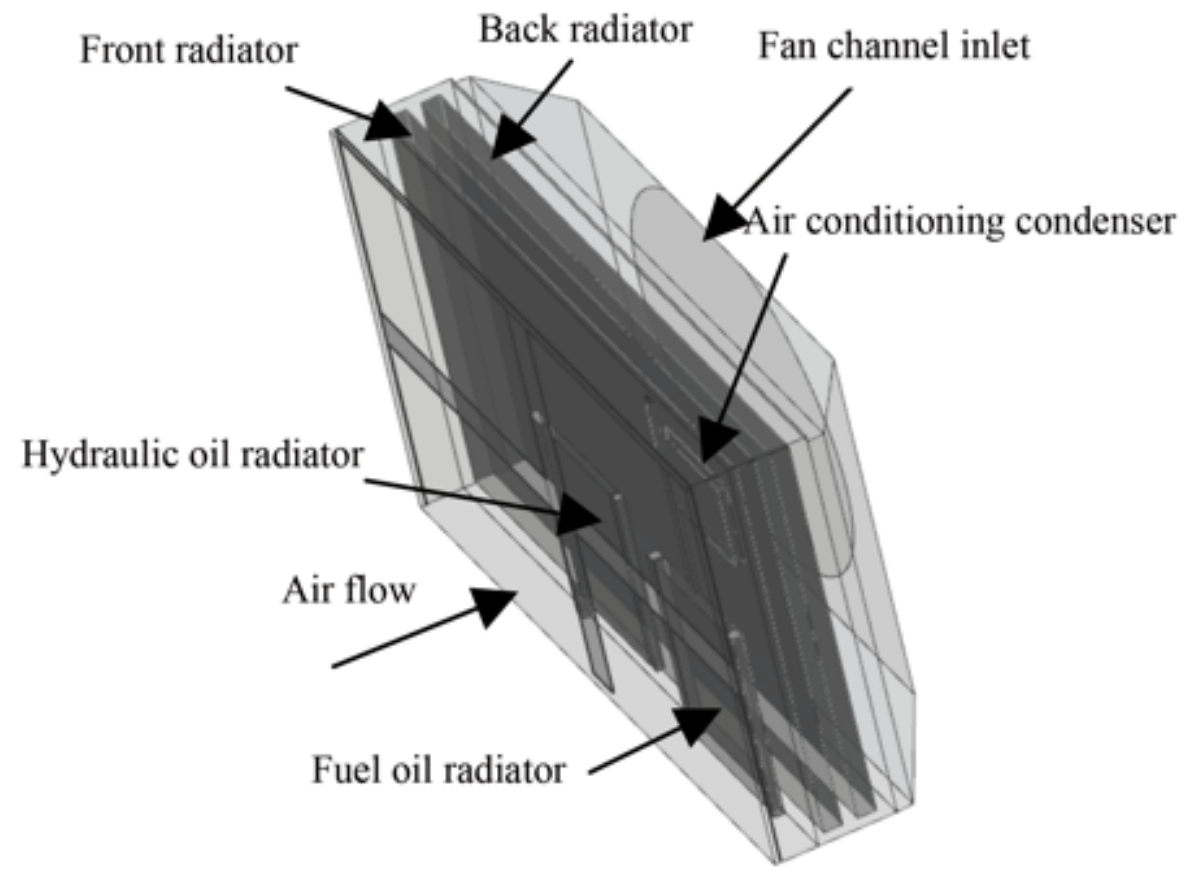

Fig. (1). Air channel structure.

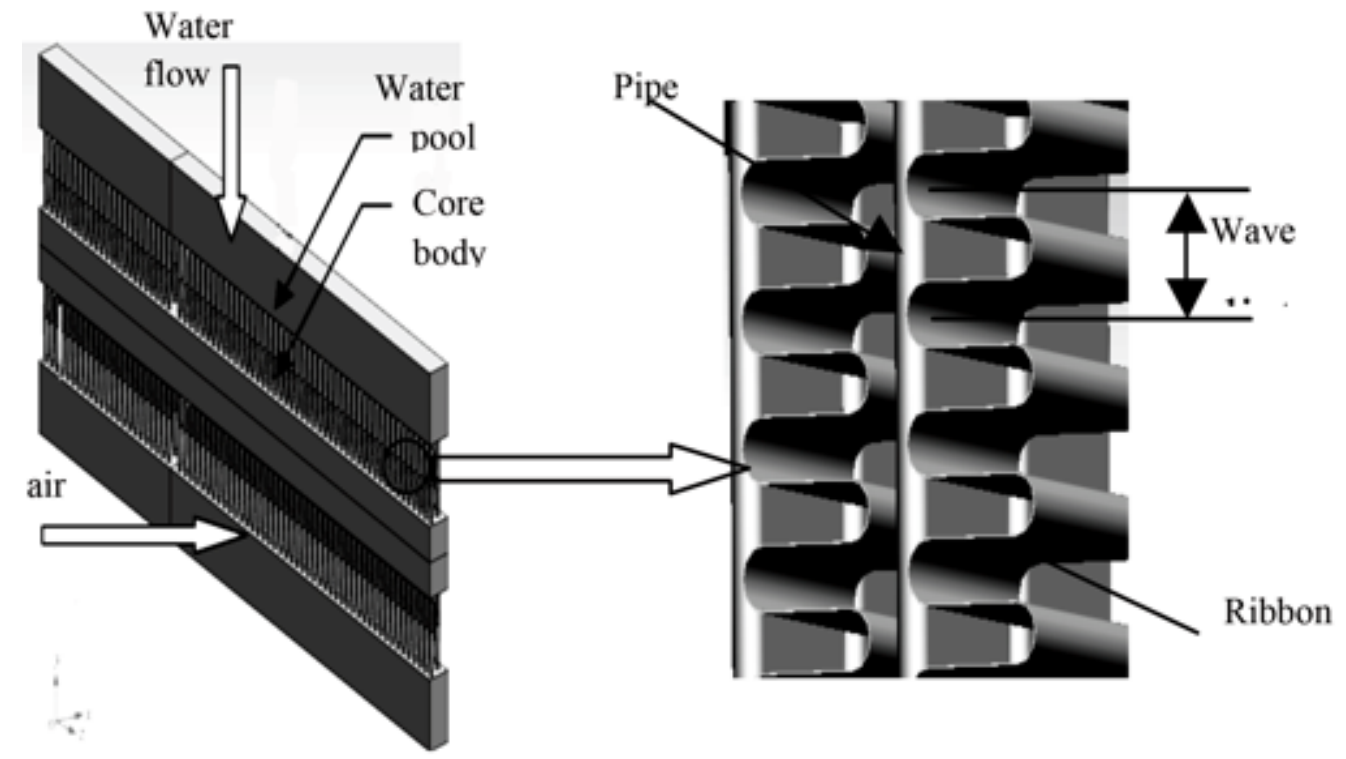

Fig. (2). Structure of ribbon-tubular radiator. 
air channel is less than 0.3 and the flow field can be viewed as a $3 \mathrm{D}$ incompressible turbulent flow. The basic physics equations of flow field and heat transfer process are continuity equation, momentum equation and energy equation $[11,12]$.

Because the turbulence quantities include the mean and fluctuating values, the momentum equation cannot be solved by N-S equations. Reynolds derived Reynolds equation (equation 1) from the incompressible flow N-S equation and with the introduction of the Reynolds stress $\rho \overline{u_{i}^{\prime} u_{j}^{\prime}}$, but Reynolds stress relations need to be added to close the Reynolds equation [13]. Since the dump truck driving speed is low, so RNG k-e turbulent model for enclosing Reynolds equation is chosen which provides an analytically-derived differential formula of effective viscosity that accounts for low-Reynolds-number effects as shown in equation 2 [11, $13,14]$.

$$
\begin{aligned}
& \frac{\partial\left(\rho u_{i}\right)}{\partial t}+\frac{\partial}{\partial x_{j}}\left(\rho u_{i} u_{j}\right)=-\frac{\partial p}{\partial x_{i}}+\frac{\partial}{\partial x_{j}}\left(\mu \frac{\partial u_{i}}{\partial x_{j}}-\rho \overline{u_{i}^{\prime} u_{j}^{\prime}}\right) \\
& d\left(\frac{\rho^{2} k}{\sqrt{\varepsilon \mu}}\right)=1.72 \frac{\hat{v}}{\sqrt{\hat{v}-1+C_{V}}} d \hat{v}
\end{aligned}
$$

In equation $2, \hat{v}=\frac{\mu_{\text {eff }}}{\mu}, C_{V}=100$.

\subsection{Multi-Scale Models Simulations and Coupling}

Because of the small structural feature sizes of the radiator core body, the radiator air-side low field simulation with the core body structure details requires a huge storage space which is difficult to achieve. But on the other hand, the radiator core body structural details are the determinants of the air flow field around it and which is also an important factor for radiator cooling performance. Therefore, the radiator unit model containing the core body structure details and the radiator whole model without the core body structure details are respectively established in this investigation and the simulation results of unit model are disposed as the characteristic data of the whole model to calculate the radiator thermal performance exactly and cheaply.

\subsubsection{Radiator Unit Model Simulations}

According to the periodical characteristic of radiator core body structure shown in Fig. (2), a cuboid zone containing one wave distance is taken as the unit model structure which is adopted in simulations for obtaining the radiator's air-side resistance characteristics and heat transfer efficiency. As shown in Fig. (3), in unit model, all geometric details of the radiator core body are contained.

The flow field is simulated by software Fluent. SIMPLE method is selected for pressure-speed coupling. The first upwind method is used firstly to obtain steady flow field and the second upwind method is applied to achieve convergence at last. In order to obtain resistance and heat transfer characteristic, flow fields of a group of air inlet velocity ( 2

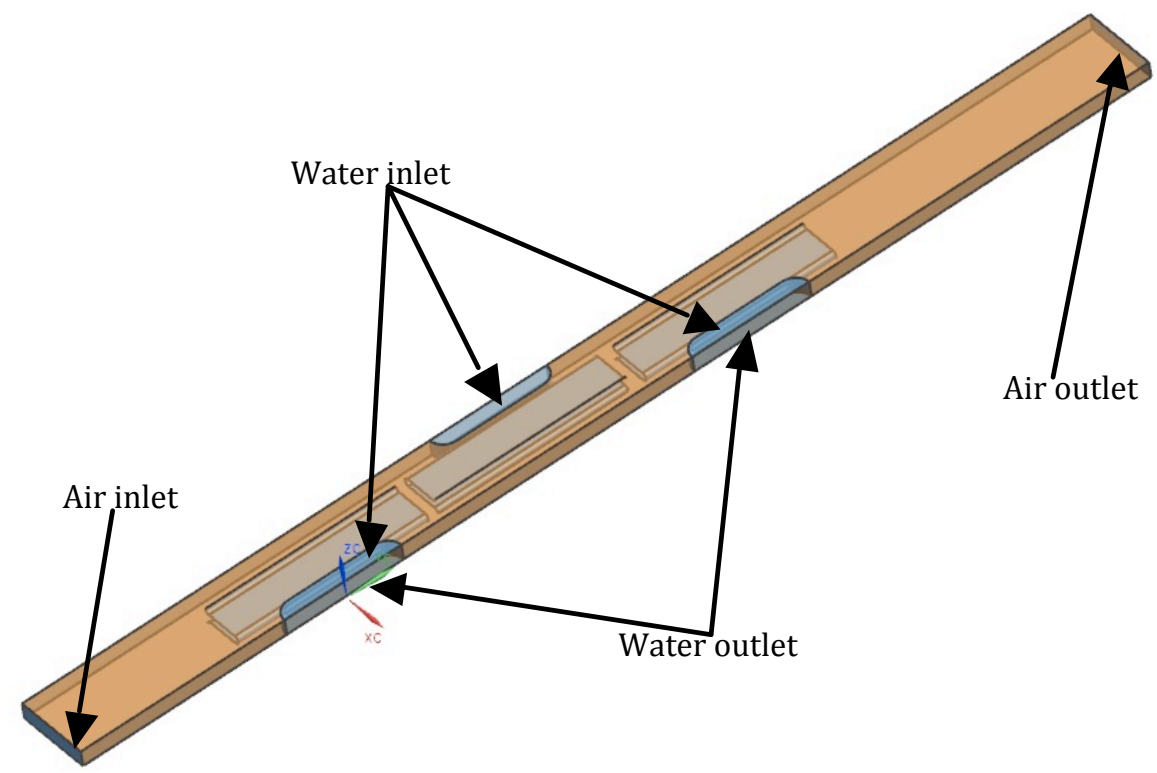

Fig. (3). Radiator unit model.

Table 1. Unit model results.

\begin{tabular}{|c|c|c|c|c|c|c|c|c|}
\hline \multicolumn{2}{|c|}{ Velocity (m/s) } & $\mathbf{2}$ & $\mathbf{4}$ & $\mathbf{6}$ & $\mathbf{8}$ & $\mathbf{1 0}$ & $\mathbf{1 2}$ & $\mathbf{1 4}$ \\
\hline \hline \multirow{2}{*}{ Front radiator } & Pressure drop(pa) & 20.5 & 53.1 & 97.0 & 152.18 & 218.4 & 295.4 & 383.1 \\
\cline { 2 - 10 } & Efficiency & 0.487 & 0.470 & 0.451 & 0.433 & 0.415 & 0.396 & 0.378 \\
\hline \multirow{2}{*}{ Back radiator } & Pressure drop(pa) & 50.2 & 87.3 & 149.1 & 232.4 & 334.2 & 451.4 & 581.1 \\
\cline { 2 - 10 } & Efficiency & 0.436 & 0.412 & 0.391 & 0.371 & 0.352 & 0.335 & 0.319 \\
\hline
\end{tabular}


$\mathrm{m} / \mathrm{s}, 4 \mathrm{~m} / \mathrm{s}, 6 \mathrm{~m} / \mathrm{s}, 8 \mathrm{~m} / \mathrm{s}, 10 \mathrm{~m} / \mathrm{s}, 12 \mathrm{~m} / \mathrm{s}, 14 \mathrm{~m} / \mathrm{s}$ ) are simulated.

After simulations, the results are processed. For the simulation results under a certain velocity, equation 3 is adopted for calculating the heat transfer efficiency and the air-side pressure drop between inlet and outlet is considered as the resistance measurement. The heat transfer efficiency and pressure drop data of the two bigger radiators are shown in Table 1 :

$\varepsilon=\frac{\left(T_{\text {outa }}-T_{\text {ina }}\right)}{\left(T_{\text {inh }}-T_{\text {ina }}\right)}$

In equation 3, $T_{\text {ina }}$ and $T_{\text {outa }}$ are respectively the air temperatures at inlet and outlet of the radiator unit model; $T_{i n h}$ is the radiator heat fluid water temperature at inlet.

\subsubsection{Radiator Whole Model Simulations}

According to the radiator structure characteristics, the whole radiator can be regarded as a one-dimensional porous medium model based on the Darcy's law and its air-side resistance characteristic is represented by the equation 4 [15]. In the right hand side of the equation 4 , the first term represents viscous losses and $\beta$ is the permeability factor; the second term represents the inertial losses, $C_{2}$ is inertial resistance factor. Two coefficients $C_{2}$ and $\beta$ are obtained by solving the equations 4 after substituting the pressure drop and velocity data in Table 1. 'Heat exchanger' model in Fluent is adopted for radiators' heat transfer simulation, and the heat transfer efficiency data in Table $\mathbf{1}$ are used for the input parameters of the 'Heat exchanger'. For further enhancing the simulation accuracy of heat transfer, the heat resource distributions of the whole radiator models are set according to the heat fluid water flow path:

$\nabla p=\frac{\mu}{\beta} v+C_{2} \frac{\rho}{2} v^{2}$

To account for the air-side flow field distribution influence on the radiator performance, the flow field in the whole air channel (shown in Fig. 1) is simulated in the radiator whole model simulation. The flow field is also simulated by software Fluent. The related sets in simulation are similar to the unit model simulations.

After simulation, the radiators' cooling powers are calculated using water inlet and outlet temperatures obtained in the radiator whole model simulation.

\subsection{Experiment and Verification}

To validate the reliability of the multi-scale models simulations coupling method, experiment of the real mining dump truck is carried out and the test sensors for radiators outlet heat water temperatures are installed as shown in Fig. (4). After the vehicle running for about one hour and 10 minutes, the radiators' inlet and outlet water temperatures are observed to be stable which are tested for the calculation of radiators' cooling power.

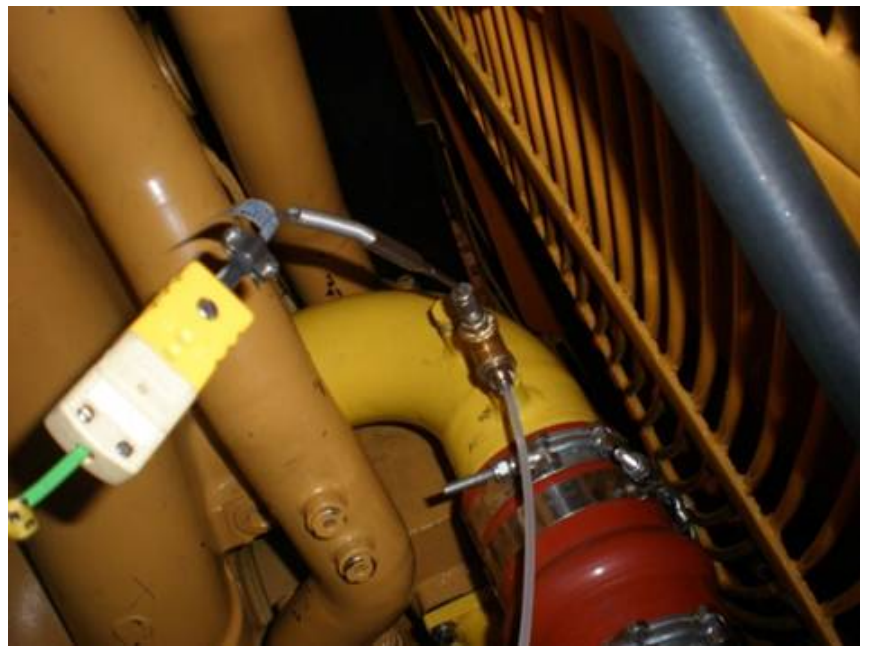

Fig. (4). Sensors installed in experiment

Table 2. Thermal powers of experiment and simulations.

\begin{tabular}{|c|c|c|}
\hline & $\boldsymbol{P}_{\text {Sim }} /(\mathbf{k W})$ & $\boldsymbol{P}_{\text {Exp }} /(\mathbf{k W})$ \\
\hline \hline Front radiator & 559.1 & 540.2 \\
\hline Back radiator & 671.8 & 643.5 \\
\hline \multicolumn{2}{|c|}{$P_{\text {Sim }}$ represents the cooling powers from simulations; $P_{\text {Exp }}$ represents the cooling powers }
\end{tabular}
from experiment.

The cooling powers from simulations and experiments are shown in Table 2 . From Table 2 , it can be seen that cooling powers from the simulations and experiment are nearly consistent and the errors of simulation values both of the front and back radiators are less than 5\% so the radiator thermal performance calculation method based on multiscale models simulations is reliable.

\section{RADIATORS' THERMAL PERFORMANCE OF TWO LAYOUT SCHEMES}

\subsection{Two Spatial Layout}

The structure and layout of the parts around radiators are the decisive factors of radiators' air-side flow field and the thermal performance. To analyze the influence of the heat source parts (the three small radiators) in front of the radiators, they are arranged in two opposing schemes for comparative analysis. As shown in Fig. (5): In scheme one, the heat source parts are situated at the center circle area which is right in the front of the rear fan channel; on the contrary, in scheme two, the parts are situated in the periphery of the center circle area.
Scheme one

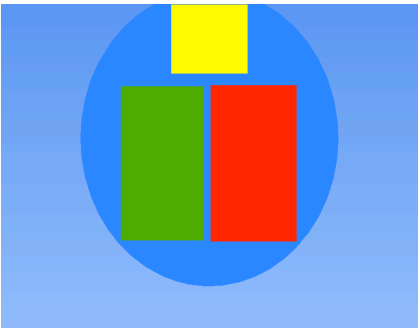

Fig. (5). Two spatial layout of the front parts.
Scheme two

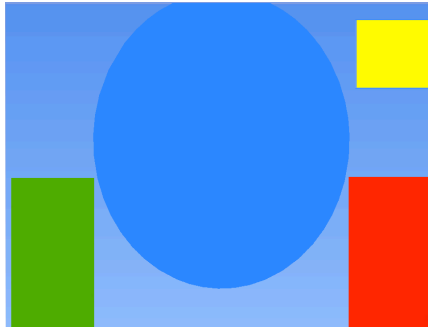


(a) Velocity

Scheme one

Scheme two

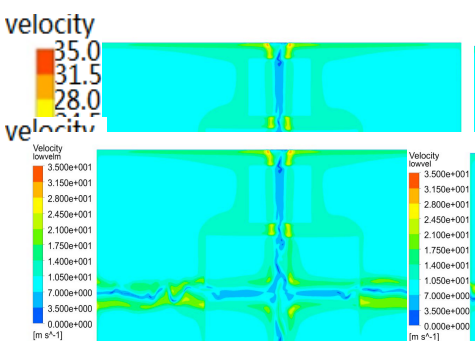

(b) Temperature

Scheme one

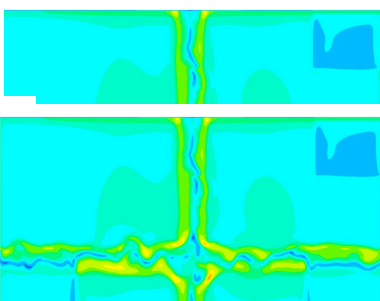

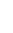

temperature
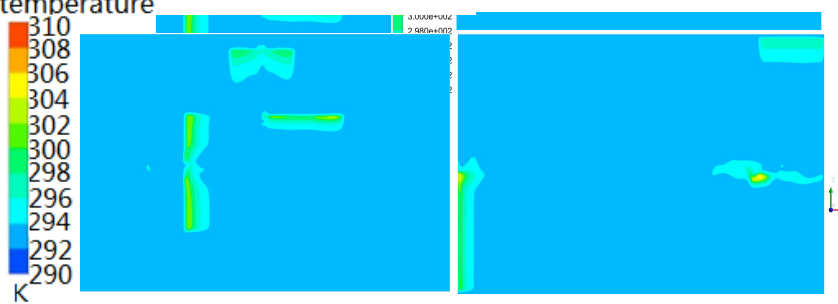

Fig. (6). Windward flow field of the front radiator.

(a) Velocity

Scheme one

Scheme two
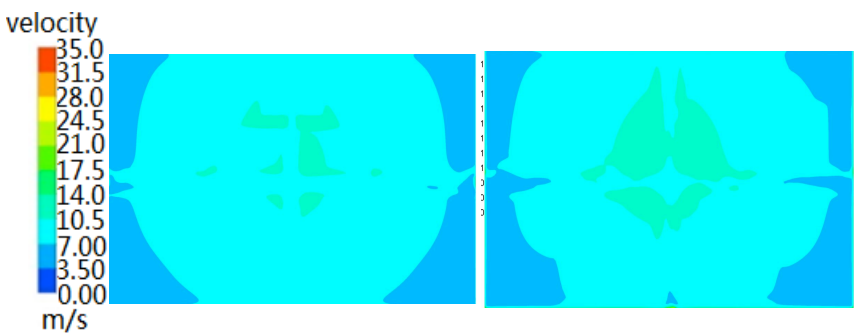

(b) Temperature

Scheme one

Scheme two

temperature

310
308
306
304
302
300
298
296
294
292
290

Fig. (7). Windward flow field of the back radiator.

\subsection{Simulation Result and Analysis}

The two radiators thermal performances of the two schemes are calculated by the simulations coupling method.
The results of windward flow fields and the cooling powers of the radiators are processed and shown in Figs. $(6,7)$ and Tables 3 and 4.

\subsubsection{Air Velocity Distribution}

As shown in Figs. $(\mathbf{6 a}, 7 \mathbf{7 a})$, because of the layout difference of the heat resource parts in front of the two radiators, the air-side flow fields of the two schemes are different. The center area of the front radiator windward is high velocity area because of the rear fan channel situation, but then in scheme one, the parts arranged at center area induce more air flow through the periphery, so the velocity difference of the center area and the periphery is weakened; on the contrary, the difference is strengthened in scheme two. So the front radiator windward velocity uniformity of scheme one is 0.019 and is more than that of scheme two, as shown in Table 3.

The windward velocity distribution of the back radiator is mainly decided by the fan because it is more nearer to the fan channel, so the velocity uniformities of the two schemes are nearly consistent.

\subsubsection{Air Temperature Distribution}

As shown in Figs. $(\mathbf{6 b}, \mathbf{7 b})$, the front radiator windward air temperature distribution of the two schemes mainly decided by the inlet air temperature distribution of the air channel are nearly consistent and temperature uniformities of the two schemes are nearly equivalent as shown in Table 3.

For the back radiator, the windward air temperature distributions of the two schemes are obviously different. This is due to its location behind the front radiator and the narrow space between the front radiator and the back radiator so the back radiator windward air temperature distribution is inevitably affected by the heat resource distribution itself and the front radiator outlet surface air temperature distribution which is the result of the heat transfer of the front radiator which is affected by several factors such as the front radiator windward air flow field, the heat resource distribution of the front radiator, the layout of the front heat source parts and so on.

As shown in Table 3, the back radiator windward air temperature difference of two schemes also results in uniformity difference of the temperature differences between air and heat fluid water, which is 0.014 .

\subsubsection{Thermal performance}

From Table 4, it can be seen that the cooling powers of the front and back radiators in the scheme one are respectively $4.1 \%$ and $6.7 \%$ which are more than that in scheme two. So scheme one is better than scheme two. Combining the flow field distribution characteristics analyzed above, it can be deduced that the more uniform air

Table 3. Uniformities of the radiators' flow field.

\begin{tabular}{|c|c|c|c|c|c|}
\hline \multirow{2}{*}{ Scheme } & \multicolumn{2}{|c|}{ Front Radiator } & \multicolumn{2}{c|}{ Back Radiator } & \multirow{2}{*}{ Temperature Difference } \\
\cline { 2 - 6 } & Velocity & Temperature & Velocity & Temperature & 0.883 \\
\hline \hline one & 0.953 & 0.994 & 0.962 & 0.972 & 0.969 \\
\hline two & 0.934 & 0.993 & 0.960 & 0.962 & 0.86 \\
\hline
\end{tabular}


velocity of the front radiator windward and the more uniform air and water temperature difference of the back radiator of the scheme one make the radiators' cooling power more than that of scheme two. So the uniformities of the windward air velocity and temperature difference between air and radiator heat fluid water are the impact factors of the radiator thermal performance. On the other hand, the uniformity difference $(0.014)$ of the two schemes of the back radiator water and air temperature difference is less than the air velocity uniformity difference $(0.019)$ of the front radiator, but compared to scheme two, the scheme one cooling power increase ratio of the back radiator is higher than that of the front radiator, so the influence of the temperature difference uniformity is more significant than air velocity uniformity.

Table 4. Radiators cooling power of two schemes.

\begin{tabular}{|c|c|c|}
\hline Scheme & $\boldsymbol{P}_{\text {Simf }} / \mathbf{k W}$ & $\boldsymbol{P}_{\text {Simb }} / \mathbf{k W}$ \\
\hline \hline One & 574.1 & 701.1 \\
\hline Two & 551.5 & 656.9 \\
\hline
\end{tabular}

$P_{\text {Simf }}$ represents the front radiator cooling powers from simulations; $P_{\text {Simb }}$ represents the back radiator cooling powers from simulations.

\section{MODIFICATION FOR RADIATORS' THERMAL PERFORMANCE}

According to the conclusions stated above, to improve the radiators' thermal performance, the front heat source parts should be moved to the center area. Due to some spatial limits of the real mining dump truck, the layout scheme of the front heat source parts is modified as shown in Fig. (8).

The radiators cooling powers of the modified scheme are obtained from the same experiment mentioned in 3.3. From Tables $\mathbf{2}$ and $\mathbf{5}$, it can be seen that as compared to the original layout, the two radiators cooling powers of the modified scheme increase and that of back radiator increases more. The overall increased value of the two radiators is $19.3 \mathrm{~kW}$.

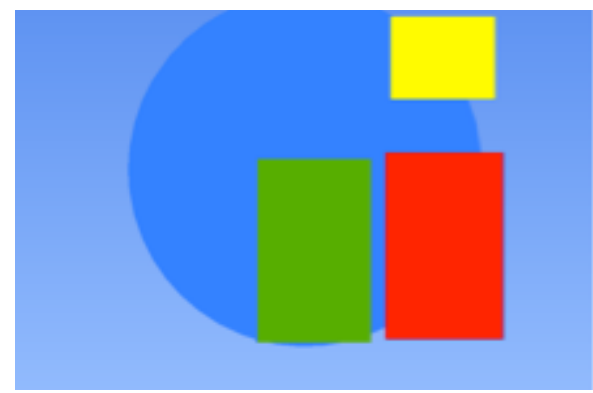

Fig. (8). Layout improvement of the front parts.

Table 5. Cooling powers of modified scheme.

\begin{tabular}{|c|c|c|}
\hline & Front Radiator & Back Radiator \\
\hline \hline$P_{\mathrm{Exp}} /(\mathrm{kW})$ & 546.3 & 656.7 \\
\hline
\end{tabular}

\section{CONCLUSION}

For the radiators of a mining dump truck, this paper adopted a thermal performance calculation method based on multi-scale models simulation coupling and the experiment values verified the reliability of the calculation method. Then, the parts layout influence on the radiator thermal performance was analyzed. At last, the layout of the heat source parts in front of the radiators was modified to improve the radiators thermal performance. Main conclusions drawn are as follows:

1. The radiator thermal performance calculation method based on simulations coupling of radiator multi-scale models considers the influence of air-side flow field distribution and the core body structure details; as compared to tested values, the errors of the calculating cooling power are less than $5 \%$, therefore, the thermal performance calculation method is reliable.

2. When the heat source parts in front of the radiators are situated right in front of the rear fan channel, the radiators' thermal performance is better; the radiator cooling power increases $19.3 \mathrm{~kW}$ after moving the heat source parts near the area right in front of the rear fan channel.

\section{CONFLICT OF INTEREST}

The authors confirm that this article content has no conflict of interest.

\section{ACKNOWLEDGEMENTS}

The original radiators' inlet and outlet water temperatures test values used in this paper were kindly provided by Senior Engineer LUO and QIU at Hunan Electric Group Co., Ltd. China. The research is funded by the China Central Treasury innovative team project (0420036017) and Natural Science Fund Project (2013HZX05) of Hunan University of technology.

\section{REFERENCES}

[1] J. Chiou, "Study of the arrangement of automobile air-conditioning condenser and engine radiator in the cooling air circuit", SAE Technical Paper 810503, 1981, doi:10.4271/810503.

[2] Z. Yang, J. Bozeman, F. Shen, and D. Turner, "CFRM concept for vehicle thermal system," SAE Technical Paper 2002-01-1207, 2002, doi:10.4271/2002-01-1207.

[3] H.J. Kim, and J. Kim, "A numerical analysis for the cooling module related to automobile air-conditioning system," Applied Thermal Engineering, vol. 28, pp. 1896-1905, 2008.

[4] T. Juan, "Investigation and assessment of factors affecting the underhood cooling air flow using CFD," SAE Technical Paper 2008-01-2658, 2008, doi:10.4271/2008-01-2658.

[5] L. Larsson, T. Wiklund, and L. Löfdahl. "Cooling performance investigation of a rear mounted cooling package for heavy vehicles," SAE Technical Paper 2011-01-0174, 2011, doi:10.4271/2011-01-0174.

[6] B. Heinzelmann, T. Indinger, N. Adams, and R. Blanke, "Experimental and Numerical Investigation of the Under Hood Flow with Heat Transfer for a Scaled Tractor-Trailer," SAE International Journal of Commercial Vehicles, vol. 5, no. 1, pp. 4256, 2012, doi:10.4271/2012-01-0107.

[7] S. Saab, J. Hetet, A. Maiboom, and F. Charbonnelle. "Impact of the underhood opening area on the drag coefficient and the thermal performance of a vehicle," SAE Technical Paper 2013-01-0869, 2013, doi:10.4271/2013-01-0869.

[8] J.N. Mao, H.X. Chen, and H. Jia, "Effect of air-side flow maldistributionon thermal-hydraulic performance of the multilouvered fin and tube heat exchanger", International Journal of Thermal Sciences, vol. 12, no. 73, pp. 46-57, 2013.

[9] H.R. Wan, X.L. Tang, and J.D. Duan, "Present status and future development of a heavy duty electrical wheels autonomous dump 
truck in mine", Automotive Research and Development, vol. 2, pp. 8-14, 2001.

[10] X.L. Wei, G.Q. Wang and S.L. Feng, "Aerodynamic characteristics about mining dump truck and the improvement of head shape", Journal of Hydrodynamics vol. 20, no. 6, pp. 713-718, 2008.

[11] B. Pope Stephen, "Turbulent flows", Cambridge University Press \& Beijing World Publishing Corporation, 2010.

[12] W.Q. Tao, "Numerical heat transfer", Xian China: Xi'an Jiaotong University Press, 1988.
[13] Z.S. Zhang, "Turbulent flows", Beijing China: National Defense Industry Press, 2002.

[14] S. Osher, and A. Sethian, "Fronts propagating with curvaturedependent speed: Algorithms based on Hamilton-Jacobi formulations", Journal of Computational Physics, vol. 79, pp. 1249, 1988.

[15] W. Liu, A.W. Fan, and M. Huang, "Heat and mass transfer theory and application of porous medium", Beijing: Science Press, 2006, 12 .

(C) Shui-Chang et al.; Licensee Bentham Open.

This is an open access article licensed under the terms of the Creative Commons Attribution Non-Commercial License (http://creativecommons.org/licenses/by-nc/4.0/) which permits unrestricted, non-commercial use, distribution and reproduction in any medium, provided the work is properly cited. 